\title{
Multi-scale modelling of the trabecular bone elastoplastic behaviour under compression loading
}

\author{
A. Jaziri ${ }^{\mathrm{a}}$, J. Rahmoun $^{\mathrm{b}}$, H. Naceur ${ }^{\mathrm{b}} *$, P. Drazetic ${ }^{\mathrm{b}}$ and E. Markiewicz \\ ${ }^{a}$ National Engineering School of Tunis, BP 37, Le Belvedere 1002, Tunis, Tunisia; ${ }^{b}$ Laboratory LAMIH, \\ University of Valenciennes, 59313 Valenciennes, France
}

\begin{abstract}
We propose a new elastoplastic damage coupled model for the modelling of trabecular bone behaviour. The damage is carried out thanks to the limit analysis based on the MCK criterion. We first present the methodology allowing the estimation of elastic anisotropic properties of porous media by means of Mori-Tanaka homogenisation scheme. Then, we present the formulation of the integrated yield criterion derived by considering trial velocity field inspired from the Eshelby inhomogeneous inclusion solution. The obtained micromechanical model is implemented via a UMAT routine within the explicit dynamic code LS-DYNA. The proposed micromechanical model has been applied successfully for the modelling of some biomechanics applications to estimate the mechanical properties of the bovine trabecular bone.
\end{abstract}

Nous proposons un nouveau modèle élastoplastique couplé à l'endommagement pour la modélisation du comportement mécanique de l'os trabéculaire. L'endommagement est prisen compte gràce à l'analyse limite basée sur le critére MCK. Nous présentons d'abord la méthodologie permettant l'estimation des propriétés élastiques anisotropes des milieux poreux parle schéma d'homogénéisation de Mori-Tanaka. Ensuite, nous présentons la formulation du critére d'écoulement intégré obtenu par un champ de vitesse test, inspiré de la solution d'inclusion Eshelby. Le modèle micromécanique est implémenté via une routine utilisateur UMAT dans le code dynamique explicite LS-DYNA. Le modèle micromécanique proposé est appliqué avec succès dans la modélisation de quelques applications en biomécanique pour estimer les propriétés mécaniques de l'os trabéculaire bovin.

Keywords: porous media; micromechanics; MCK criterion; trabecular bone; homogenisation.

Mots-clés: Milieux poreux; micromécanique; critère MCK; os trabéculaire; homogénéisation.

\section{Introduction}

The investigation of bone tissue as a material is an important topic in biomechanics. Motivated by clinical practice, a large amount of mechanical data has been collected for bovine and human bone with testing method conventionally used for engineering materials. After the introduction of artificial orthopaedic devices, the complex problem of bone implant interaction emerged and recalled the importance of the interrelations between biological and mechanical properties.

The mechanical behaviour of trabecular bone has been extensively studied in the literature as it is one of the main constituents of the bone tissue. Enclosed in compact bone at the end of epiphyses or in the core of flat and small bones, trabecular bone is subjected to multi-axial strains and stresses during physiological loading. Compressive mechanical testing represents

*Corresponding author. Email: hakim.naceur@univ-valenciennes.fr 
an established method for evaluating the mechanical competence of trabecular bone, due to its simplicity and its resemblance to the natural deformation (Fyhrie \& Schaffler, 1994). In compression, the mechanical behaviour of cortical and trabecular bone presents distinct characteristics. Cortical bone is quasi-brittle, while the mechanical behaviour of trabecular bone is typical of an elastoplastic cellular material (Gibson \& Ashby, 1997; Hayes \& Carter, 1976). For both types of bone under compressive monotonic loading, the tangent stiffness decreases already before the maximum force is reached. For trabecular bone, a smooth and gradual reduction of the stress (softening) then occurs until a local minimum, followed by rehardening (Gibson \& Ashby, 1997). In studying this mechanical response, uniaxial mechanical compression tests were widely used to assess stiffness, strength, creep and fatigue properties of trabecular bone (Keaveny, Morgan, Niebur, \& Yeh, 2001; Keaveny, Pinilla, Crawford, Kopperdahl, \& Lou, 1997; Kosmopoulos, Schizas, \& Keller, 2008; Rincón-Kohli, 2003). Especially, Kefalas and Eftaxiopoulos (2012) investigated experimentally the mechanical response of bovine trabecular bone subjected to uniaxial compression or tension under large strains was established. It has been revealed the three stages of the constitutive response, namely the initial increasing and softening branches at moderate strains, the plateau region at large strains and the hardening part at very large strains under compaction.

However, for many years, the differences in mechanical properties of trabecular bone were explained by volume fraction (Carter \& Hayes, 1977; Rice, Cowin, \& Bowman, 1988) and most of the relevant studies have been confined to one or few skeletal sites. It has been shown that the mechanical properties of trabecular bone are influenced by architectural anisotropy (Goulet et al., 1994; Hodgskinson \& Currey, 1990). In order to describe the extent of anisotropy, the method of mean intercept length (MIL) has been introduced on planar sections (Whitehouse, 1974) and extended to 3D where its distribution was characterised by a second rank fabric tensor (Harrigan \& Mann, 1984). In the pioneering work of Cowin (1985), a general relationship between volume fraction, fabric, which is a second-order tensor approximation of architectural anisotropy, and elastic properties was established.

From a modelling point of view, the relationship between morphology of spongy bones to the mechanical properties and failure mechanism can be assessed through computational means. The constitutive law, which defines the relation between stresses and strains, is incorporated in the FE method to model global behaviour. Most studies have used simple constitutive relations, for example linear elasticity with isotropic or transverse isotropic symmetry and the onedimensional model of Fondrk, Bahniuk, and Davy (1999a) unsuitable for three-dimensional finite element simulations. So far, only little effort has been devoted to the development of inelastic contitutive models specific to bone (Fondrk, Bahniuk, and Davy, 1999b; Zysset \& Curnier, 1996). In a consequent work, Gupta, Bayraktar, Fox, Keaveny, and Papadopoulos (2007) developed an infinitesimal plasticity-like model with isotropic kinematic hardening to describe yielding of trabecular bone at the continuum level. Based on the porous structure of the trabecular tissue, Gibson and Ashby (1997) have presented a multilinear parametric model up to compaction. In addition, Zysset and Curnier (1996) have proposed a three-dimensional constitutive law which describes anisotropic elasticity and the rate-independent degradation in mechanical properties from the growth of cracks or voids in the trabecular tissue. In Thurner et al. (2006), the authors have investigated the failure mechanism in trabecular bone specimens under compression. They have reported that trabecular bone often fails due to delamination, caused by microcracking and cracking, providing a mechanism for energy dissipation while conserving trabecular bone architecture. Recently, Charlebois, Jirásek, Philippe, and Zysset (2010) have proposed a constitutive law which captures the hardening-softening response of trabecular bone in compression and couples damage and plasticity with a non-local formulation (Grassl \& Jirásek, 2006; Hansen \& Schreyer, 1994; Lubarda \& Krajcinovic, 1995; Maugin, 1992; Zysset \& Curnier, 1996). 
In this paper, we propose a micromechanical elastoplastic model of the bone behaviour in view of an integration of bone damage for the simulation of necking and rupture initiation. The determination of elastic properties has been made by using the Mori-Tanaka scheme and experimental measurements of anisotropy by microtomography techniques. The non-linear behaviour is performed by a coupling between plasticity and damage on a microscopic scale. The damage has been carried out by using a limit analysis approach based on the MCK criterion (Monchiet, Charkaluk Kondo, \& Kondo, 2007, 2011). The derived yield function is performed by the consideration of new trial velocity fields inspired from the Eshelby $(1957,1959)$ exterior point solution to inclusions problem in which the eigen-strains are unknown. The obtained elastoplastic damage behaviour law is implemented via a user material routine UMAT within the explicit dynamic commercial code LS-DYNA (Hallquist, 2001) for the prediction of the global response of porous media. Finally, we present some applications in structural mechanics to study a cylindrical tensile specimen and in biological tissues to estimate the mechanical properties of the bovine trabecular bone. The adaptation of this model to biological tissues is made by considering a basic cell in conformity with the trabecular bone microstructure.

\section{Formulation of the micromechanical model}

\subsection{Homogenisation scheme for elastic anisotropic properties}

Micromechanical analysis provides links between macroscopic properties of material and its microstructure. In common homogenisation methods, the material properties are assumed to be homogeneous but unknown at macroscopic scale, whereas heterogeneous but known at microscopic scale. The main task is to find the homogeneous material properties at macroscopic scale based on the available information at microscopic scale. For this purpose, and as classically, a representative elementary volume (REV) is generally adopted to represent the idealised microstructure of material. In the present study, the REV is composed of an isotropic linear elastic solid matrix with elastic stiffness tensor $\mathbb{C}^{(\mathrm{m})}$ weakened by a random distribution of ellipsoidal shaped pores made up of phases $r(r=1, \ldots, N)$ with the elasticity tensor $\mathbb{C}^{(\mathrm{r})}$. The inclusions of phase $r$ are taken to be identical in shape and orientation with the normal $\underline{n}$, radius $a$ and the average half-opening $c$.

Based on the matrix-inclusion problems (Eshelby, 1957; Laws \& McLaughlin, 1979) and the Mori-Tanaka scheme (Mori \& Tanaka, 1973), an estimate for the homogenised elastic stiffness tensor of the bone material reads as (Rahmoun, Chaari, Markiewicz, \& Drazetic, 2009):

$$
\mathbb{C}^{\text {hom }}=\mathbb{C}^{(\mathrm{m})}+\sum_{r=1}^{N} f^{(\mathrm{r})}\left(\mathbb{C}^{(\mathrm{r})}-\mathbb{C}^{(\mathrm{m})}\right): \mathbb{A}_{\mathrm{w}}^{(\mathrm{r})}:\left(f^{(\mathrm{m})} \rrbracket+\sum_{s=1}^{N} f^{(\mathrm{s})} \mathbb{A}_{\mathrm{w}}^{(\mathrm{s})}\right)^{-1}
$$

where $f^{(\mathrm{m})}$ and $f^{(\mathrm{r})}$ are, respectively, the volume fraction of matrix and inclusions and where the two sums are taken over all phases of the heterogeneous material in the REV. $A_{w}^{(r)}$ denotes the strain concentration tensor associated with the $r$ th phase family of inclusions of aspect ratio $w=\frac{c}{a}$, which writes:

$$
\mathbb{A}_{\mathrm{w}}^{(\mathrm{r})}=\left[\mathbb{\square}+\mathbb{P}_{\mathrm{w}}^{(\mathrm{r})}:\left(\mathbb{C}^{(\mathrm{r})}-\mathbb{C}^{(\mathrm{m})}\right)\right]^{-1}
$$

and $\mathbb{P}_{\mathrm{w}}^{(\mathrm{r})}$ is the Hill tensor (Hill, 1965). This tensor depends on the shape and orientation of the r-th family of inclusions $\mathbb{C}^{(\mathrm{r})}$ (considered here as spheroid) and the elastic stiffness of the reference medium $\mathbb{C}^{(\mathrm{m})}$. 
Finally, as in the previous work (Rahmoun et al., 2009), the formalism of stiffness tensor (1) is coupled with experimental measurements of architectural anisotropy obtained from X-ray micro tomography and the MIL method (Harrigan \& Mann, 1984; Whitehouse, 1974), which will, in turn, be used to calculate the porosity.

The necessary detail can be found in Rahmoun et al. (2009), and only a summary of the main results of the model will be presented here.

\subsection{The constitutive plastic-damage model based on the MCK criterion}

In this section, we present an extension of the micromechanical linear model for the case of non-linear behaviour. The new model is based on a coupling between plasticity and damage on a microscopic scale. The damage has been carried out by using the framework of limit analysis of Gurson (1977) and its evolutions, in particular, the recent work of Monchiet et al. (2007, 2011), called MCK criterion. In this approach, the considered domain is composed of a spherical cavity with the radius $a$ subjected to a uniform eigen-strain rate $d^{*}$ and embedded in a spherical cell with the radius $b$. The spherical frame (coordinates $r, \varphi, \theta$ ) is considered to describe the studied cell. The trial velocity field in the matrix, $\underline{v}$, required for the limit analysis of the hollow sphere (Gurson, 1977), classically takes the following general form:

$$
\underline{v}=A \cdot \underline{x}+\underline{v}^{\mathrm{E}}
$$

in which $A . \underline{x}$ is associated to a uniform strain rate $A$ and $\underline{v}^{\mathrm{E}}$ is a heterogeneous field which corresponds to the expansion of the cavity. The matrix incompressibility implies that $\operatorname{tr}(A)=0$ and $\operatorname{div}\left(\underline{v}^{\mathrm{E}}\right)$.

From the exterior-point Eshelby solution (Eshelby, 1959; Kachanov, Shafiro, Tsukrov, \& Hanbook, 2003; Mura, 1987), a more refined velocity field $\underline{v}^{\mathrm{E}}$, in the particular case of a spherical inclusion, can be given by:

$$
\underline{v}^{\mathrm{E}}=\sum_{r=1}^{r=6} \underline{v}^{\mathrm{r}} d_{\mathrm{r}}^{*}
$$

where $d_{\mathrm{r}}^{*}$ is related to the eigen-strain in the inhomogeneity $d^{*}$. The microscopic plastic strain rate $d$ derived from the velocity field (3) is defined by:

$$
d=A+d^{\mathrm{E}}
$$

with $d^{\mathrm{E}}=\nabla_{\mathrm{s}} \underline{v}^{\mathrm{E}}$, the symmetric part of the velocity gradient associated to $\underline{v}^{\mathrm{E}}$ (Monchiet et al., 2007, 2011). The microscopic dissipation $\pi(d)=d_{0} d_{\mathrm{eq}}$, where $d_{\mathrm{eq}}=\sqrt{\frac{2}{3} d^{\prime}: d^{\prime}}$ is the equivalent plastic strain rate defined by (see Monchiet et al., 2007, 2011):

$$
d_{\mathrm{eq}}^{2}=A_{\mathrm{eq}}^{2}+2 \sum_{r=1}^{r=6} d_{\mathrm{r}}^{*} A: d^{\mathrm{r}}+\sum_{r=1}^{r=6} \sum_{s=1}^{s=6} d_{\mathrm{r}}^{*} d_{\mathrm{s}}^{*} d^{\mathrm{r}}: d^{\mathrm{s}}
$$

and $d^{\prime}$ is the deviatoric part of strain tensor $d$. Then, in agreement with Hill-Mandel lemma, the macroscopic strain rate $D$ is related to the local strain rate, $d$, by the average rule:

$$
\frac{1}{|\Omega|} \int_{\Omega} d d V
$$

where $|\Omega|$ is the volume of the studied cell (matrix + void). 
The macroscopic plastic dissipation will be obtained after a minimisation procedure on the remaining unknown parameters $d^{* *}$ :

$$
\Pi(D)=\min _{\left(d^{*}\right)^{\prime}}\left[\tilde{\Pi}\left(D,\left(d^{*}\right)^{\prime}\right)\right] \quad \text { with } \quad \min _{\left(d^{*}\right)^{\prime}}\left[\tilde{\Pi}\left(D,\left(d^{*}\right)^{\prime}\right)\right]=\frac{\sigma_{0}}{|\Omega|} \int_{\Omega-w} d d V
$$

The yield surface, related the macroscopic dissipation, is performed by:

$$
\Sigma=\frac{\partial \Pi}{\partial D}
$$

Finally, following the limit analysis method of Gurson (1977), the approximate expression of the macroscopic yield function is obtained as (Monchiet et al., 2007, 2011):

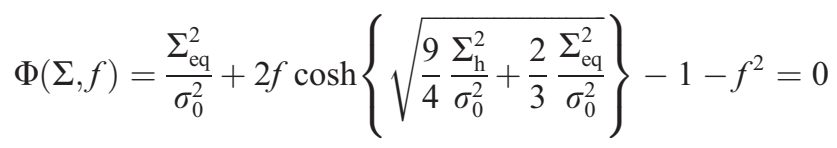

where $\Sigma_{\mathrm{h}}$ denotes the hydrostatic stress and $f$ the material porosity. $\Sigma_{\text {eq }}$ is the macroscopic von-Mises equivalent stress.

\section{Numerical integration of the plastic-damage model}

As stated before, we aim at formulating and implementing the MCK criterion. The implementation has been carried out within the explicit dynamic code LS-DYNA via the user-routine UMAT. For comparison purpose, the GTN model (which is a standard modification of the Gurson model) is also considered.

We introduce a hardening variable in the macroscopic yield function given by (10) by following a classical approach through the yield stress in the matrix $\bar{\sigma}$ :

$$
\Phi(\Sigma, \bar{\sigma}, f)=\frac{\Sigma_{\text {eq }}^{2}}{\bar{\sigma}^{2}}+2 f \cosh \left\{\sqrt{\frac{9}{4} \frac{\Sigma_{\mathrm{h}}^{2}}{\bar{\sigma}^{2}}+\frac{2}{3} \frac{\Sigma_{\text {eq }}^{2}}{\bar{\sigma}^{2}}}\right\}-1-f^{2}=0
$$

The macroscopic plastic flow rule obtained by upscaling, according to normality rule, is given by:

$$
\dot{E}^{\mathrm{p}}=\dot{\lambda} \frac{\partial \Phi}{\partial \Sigma}
$$

in which $\dot{\lambda}$ is the plastic multiplier. Taking into account the incompressibility of the matrix, the porosity evolution law which characterises the damage growth, reads:

$$
\dot{f}=3(1-f) \dot{E}_{\mathrm{m}}^{\mathrm{p}}+A \dot{\bar{\varepsilon}}_{\mathrm{m}}^{\mathrm{p}}
$$

where $E_{\mathrm{m}}^{\mathrm{p}}$ is the volumetric plastic strain and where $\vec{\epsilon}_{\mathrm{m}}^{\mathrm{p}}$ is the average volumetric plastic strain in the matrix. The therm A reads:

$$
A=\frac{f_{\mathrm{N}}}{s_{\mathrm{N}} \sqrt{2 \pi}} \exp \left\{-\frac{1}{2}\left(\frac{\bar{\varepsilon}_{\mathrm{m}}^{\mathrm{p}}-\varepsilon_{\mathrm{N}}}{s_{\mathrm{N}}}\right)\right\}
$$


in which $\varepsilon_{\mathrm{N}}$ is the mean normal distribution of the nucleation strain, $s_{\mathrm{N}}$ is a standard deviation and $f_{\mathrm{N}}$ is the nucleates voids volume fraction. Therefore, the evolution of the yield stress in the matrix is given by (see for instance Gurson, 1977):

$$
(1-f) \bar{\sigma} \dot{\bar{\varepsilon}}=\Sigma: \dot{E}^{\mathrm{p}}
$$

Finally, we adopt this simple coalescence model described by Tvergaard and Needleman (1984) in order to consider the effect of void coalescence in the numerical implementation:

$$
f^{*}=\left\{\begin{array}{lll}
f & \text { if } & f \leq f_{\mathrm{c}} \\
f_{\mathrm{c}}+\delta\left(f-f_{\mathrm{c}}\right) & \text { if } & f>f_{\mathrm{c}}
\end{array}\right.
$$

where $f_{\mathrm{c}}$ represents the porosity at the onset of coalescence and $\delta=\left(f_{\mathrm{U}}-f_{\mathrm{c}}\right)\left(f_{\mathrm{F}}-f_{\mathrm{c}}\right)$ is a factor describing the acceleration of the material degradation during coalescence. $f_{\mathrm{F}}$ and $f_{\mathrm{U}}$ represent the porosity and the value of $f^{*}$ at failure, respectively.

The stress increment calculation is based on the return mapping algorithm, which includes two steps.

First an elastic prediction of stress is done:

$$
\Sigma_{n+1}=\mathbb{C}^{\text {hom }}: E_{n+1}^{\mathrm{e}}=\mathbb{C}^{\text {hom }}:\left(E_{n+1}-E_{n+1}^{\mathrm{p}}\right)=\Sigma^{\text {pred }}-\mathbb{C}: \Delta E^{\mathrm{p}}
$$

where $\mathbb{C}^{\text {hom }}$ was determined by (1) and where $\Sigma^{\text {pred }}$ is the elastic predictor expressed as: $\Sigma^{\text {pred }}=\mathbb{C}^{\text {hom }}: \Delta E$.

Then a plastic correction is used, we compute $\frac{\partial \Phi}{\partial \Sigma^{\text {pred }}}$ by using Equation (12) and we obtain the following expressions for the deviatoric part $\left(\Delta E^{\mathrm{p}}\right)^{\prime}$ and the hydrostatic part $\Delta \Sigma_{\mathrm{m}}^{\mathrm{p}}$ :

$$
\left\{\begin{array}{l}
\left(\Delta E^{\mathrm{p}}\right)^{\prime}=\Delta \lambda\left(\frac{3}{\bar{\sigma}^{2}}+\frac{2 f}{\bar{\sigma}^{2}} \frac{\sinh \left(R_{0}\right)}{R_{0}}\right)\left(\Delta \Sigma^{\mathrm{pred}}\right)^{\prime} \\
\Delta \Sigma_{\mathrm{m}}^{\mathrm{p}}=\Delta \lambda \frac{3}{2} \frac{f \Sigma_{\mathrm{m}}^{\mathrm{pred}}}{\bar{\sigma}^{2}} \frac{\sinh \left(R_{0}\right)}{R_{0}}
\end{array}\right.
$$

where $R_{0}=\sqrt{\frac{9}{4} \frac{\left(\sum_{\mathrm{m}}^{\text {pred }}\right)^{2}}{\bar{\sigma}^{2}}+\frac{2}{3} \frac{\left(\sum_{\mathrm{eq}}^{\mathrm{pred}}\right)^{2}}{\bar{\sigma}^{2}}}$.

Then, we have:

$$
\Delta \lambda=\frac{\bar{\sigma}^{2} \Delta E_{\mathrm{eq}}^{\mathrm{p}}}{\Sigma_{\mathrm{eq}}^{\mathrm{pred}}} \frac{1}{2+\frac{4 f}{3} \frac{\sinh \left(R_{0}\right)}{R_{0}}}
$$

Finally, the consistency condition $\dot{\Phi}=0$ implies to the expression of $\Delta E_{\mathrm{eq}}^{\mathrm{p}}$ and then to the plastic correction:

$$
\Delta E^{\mathrm{p}}=\Delta \lambda \frac{\partial \Phi}{\Sigma^{\mathrm{pred}}}
$$




\section{Finite element formulation}

\subsection{Kinematics}

Now we will recall the formulation of the eight-node solid-shell element. With respect to nodal designation (Figure 1), the coordinate vector $\mathbf{x}$ and the displacement vector $\mathbf{u}$ of the element are (Hannachi, Naceur, \& Batoz, 2007):

$$
\begin{aligned}
\mathbf{x}=\tilde{\mathbf{x}}(\xi, \eta) & +\frac{\zeta}{2} \mathbf{V}(\xi, \eta)=\sum_{i=1}^{4} N_{i}(\xi, \eta)\left(\frac{1-\zeta}{2} \mathbf{x}_{i}^{-}+\frac{1+\zeta}{2} \mathbf{x}_{i}^{+}\right) \\
\mathbf{u} & =\sum_{i=1}^{4} N_{i}(\xi, \eta)\left(\frac{1-\zeta}{2} \mathbf{u}_{i}^{-}+\frac{1+\zeta}{2} \mathbf{u}_{i}^{+}\right)
\end{aligned}
$$

where $\tilde{\mathbf{x}}$ is the position vector at the mid-surface, $\hat{h}$ and $\hat{\mathbf{n}}$ are the average thickness and the normal of the sheet, respectively, $N_{i}(\xi, \eta)$ are the two-dimensional eight-node Lagrangian interpolation functions, $\mathbf{x}_{i}^{-}, \mathbf{u}_{i}^{-}$and $\mathbf{x}_{i}^{+}, \mathbf{u}_{i}^{+}$are, respectively, the coordinate and displacement vectors of the $i^{\text {th }}$ node on the bottom and top shell surfaces (Figure 1).

In this work, linear, isoparametric solid-shell elements are used with bilinear interpolation in membrane and linear interpolation in thickness direction. More details regarding the kinematics of solid-shell elements may be found in Hannachi et al. (2007).

\subsection{Variational formulation}

In order to deal with the several lockings separately, one needs to separate the expression of virtual internal work by uncoupling the membrane/bending, transverse/thickness and shearing (Hannachi et al., 2007).

$$
\begin{gathered}
W_{\text {int }}=\sum_{e=1}^{\text {nelt }} W_{\text {int }}^{\mathrm{e}} ; \quad W_{\text {int }}^{\mathrm{e}}=W_{\text {int }}^{\mathrm{mb}}+W_{\text {int }}^{\mathrm{tr}}+W_{\text {int }}^{\mathrm{sh}} \\
W_{\mathrm{int}}^{\mathrm{mb}}=\int_{V^{0}} \delta \mathbf{E}_{\mathrm{mb}} \mathbf{S}_{\mathrm{mb}} \mathrm{d} V=\delta \mathbf{u}^{T} \mathbf{f}_{\mathrm{int}}^{\mathrm{mb}} ; \quad \mathbf{f}_{\mathrm{int}}^{\mathrm{mb}}=\int_{V^{0}} \mathbf{B}_{\mathrm{mb}}^{T} \mathbf{S}_{\mathrm{mb}} d V
\end{gathered}
$$

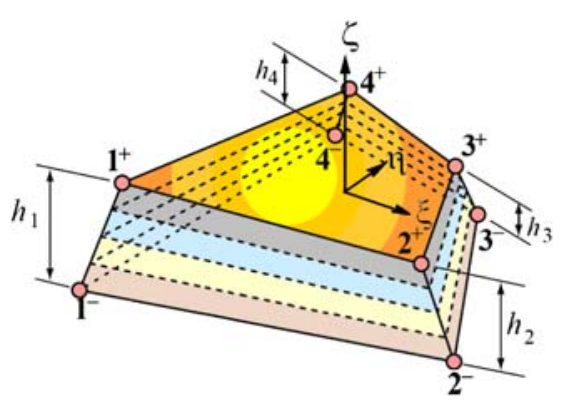

Figure 1. Eight-node solid-shell element. 


$$
\begin{aligned}
W_{\mathrm{int}}^{\mathrm{tr}}=\int_{V^{0}} \delta \mathbf{E}_{\mathrm{tr}} \mathbf{S}_{\mathrm{tr}} \mathrm{d} V=\delta \mathbf{u}^{T} \mathbf{f}_{\mathrm{int}}^{\mathrm{tr}} ; & \mathbf{f}_{\mathrm{int}}^{\mathrm{tr}}=\int_{V^{0}} \mathbf{B}_{\mathrm{tr}}^{T} \mathbf{S}_{\mathrm{tr}} \mathrm{d} V \\
W_{\mathrm{int}}^{\mathrm{sh}}=\int_{V^{0}} \delta \mathbf{E}_{\mathrm{sh}} \mathbf{S}_{\mathrm{sh}} \mathrm{d} V=\delta \mathbf{u}^{T} \mathbf{f}_{\mathrm{int}}^{\mathrm{sh}} ; & \mathbf{f}_{\mathrm{int}}^{\mathrm{sh}}=\int_{V^{0}} \mathbf{B}_{\mathrm{sh}}^{T} \mathbf{S}_{\mathrm{sh}} \mathrm{d} V
\end{aligned}
$$

with $\mathbf{E}$ the Green-Lagrange strain tensor split into three components $\mathbf{E}_{\mathrm{mb}}=\left\langle E_{11} E_{22} 2 E_{12}\right\rangle$,

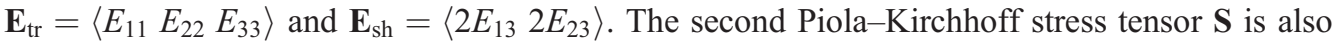
decomposed into three parts $\mathbf{S}_{\mathrm{mb}}=\left\langle S_{11} S_{22} S_{12}\right\rangle, \mathbf{S}_{\mathrm{tr}}=\left\langle S_{11} S_{22} S_{33}\right\rangle$ and $\mathbf{S}_{\mathrm{sh}}=\left\langle S_{13} S_{23}\right\rangle$. The internal force vector at the element level is then given by:

$$
\mathbf{f}_{\text {int }}^{\mathrm{u}}=\mathbf{f}_{\text {int }}^{\mathrm{mb}}+\mathbf{f}_{\text {int }}^{\mathrm{tr}}+\mathbf{f}_{\text {int }}^{\mathrm{sh}}
$$

A solid-shell element formulated using Equations (24-26) with standard integration based on a $2 \times 2$ Gauss schema in the in-plane of the shell element will fail because of numerous locking phenomena.

\subsubsection{Remedies for shear locking}

An effective method of resolving shear locking is the assumed natural strain (ANS) method in which the natural transverse shear strains are sampled and then interpolated at some discrete element points with a specific order.

The transverse shear strains $E_{\xi \zeta}$ and $E_{\eta \zeta}$ are calculated according to the average surface plan $(\zeta=0)$, assuming that they vary linearly, and are function of $E_{\xi}$ and $E_{\eta}$ at the mid-side points (Figure 2):

$$
E_{\xi \zeta}^{\mathrm{ANS}}=\frac{1-\eta}{2} E_{\xi}^{\mathrm{A} 1}+\frac{1+\eta}{2} E_{\xi}^{\mathrm{A} 2} ; \quad E_{\eta \zeta}^{\mathrm{ANS}}=\frac{1-\xi}{2} E_{\eta}^{\mathrm{B} 1}+\frac{1+\xi}{2} E_{\eta}^{\mathrm{B} 2}
$$

\subsubsection{Remedies for trapezoidal locking}

Similar to shear locking, trapezoidal locking occurs when lower order elements such as eightnode hexahedral elements are used to model curved shells, so that their cross sections assume the trapezoidal shape, these excessive number of sampled thickness strains can be reduced by

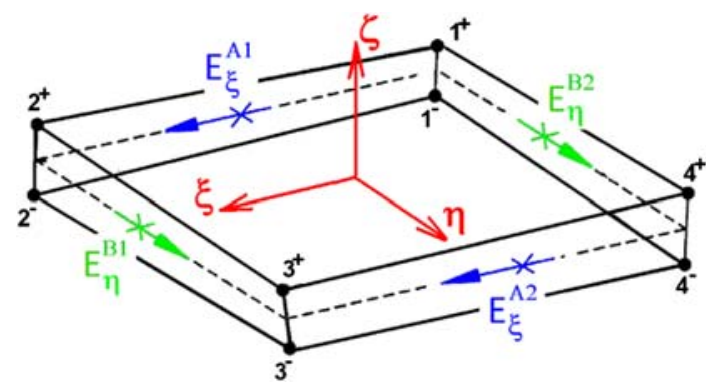

Figure 2. Shear locking treatment using ANS method. 


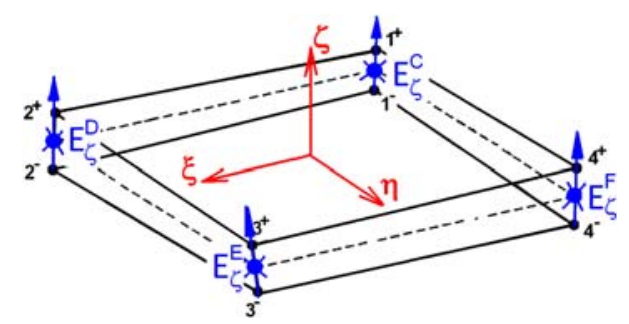

Figure 3. Trapezoidal locking treatment using ANS method.

using a bilinear interpolation of the transverse normal strains sampled at the four corners of the element mid-surface, namely (Figure 3)

$$
E_{\zeta \zeta}^{\mathrm{ANS}}=\sum_{i=1}^{4} N_{i}(\xi, \eta) E_{\zeta \zeta}\left(\xi_{i}, \eta_{i}\right)
$$

\subsubsection{Remedies for volumetric locking}

Material locking is controlled by a material parameter, the Poisson ratio $v$.

Poisson's ratio coupling requires the thickness strain to be a linear function of $\zeta$. Because our solid-shell element has only two layers, as a consequence, the thickness strain does not vary with $\zeta$; thus, the element fail in reproducing the plane-stress condition.

$$
\tilde{E}_{\zeta \zeta}^{\mathrm{EAS}}=E_{\zeta \zeta}^{\mathrm{ANS}}+\alpha \zeta t_{33}
$$

where $\alpha$ represents the seventh independent internal parameter which will be eliminated by special condensation technique at the element level, $t_{33}$ is required for transformation to the local element coordinates. An additional condition has now to be satisfied locally, leading to the increment of the additional degrees of freedom.

$$
\frac{\partial W_{\text {int }}}{\partial \mathbf{u}} \Delta \mathbf{u}+\frac{\partial W_{\text {int }}}{\partial \alpha} \Delta \alpha=-W_{\text {int }}(\mathbf{u}, \alpha)
$$

On an element level, the internal virtual work can now be computed with the compatible and the enhanced strains as in Equation (30).

$$
\begin{aligned}
W_{\mathrm{int}} & =\sum_{i=1}^{\mathrm{nl}} \int_{\xi} \int_{\eta} \int_{\zeta_{i}}^{\zeta_{i+1}}\left(\delta \mathbf{E}_{\mathrm{mb}} \mathbf{S}_{\mathrm{mb}}+\delta \mathbf{E}_{\mathrm{tr}}^{\mathrm{EAS}} \mathbf{S}_{\mathrm{tr}}+\delta \mathbf{E}_{\mathrm{sh}}^{\mathrm{ANS}} \mathbf{S}_{\mathrm{sh}}\right) J \mathrm{~d} \xi \mathrm{d} \eta \mathrm{d} \zeta \\
& =\delta \mathbf{u}^{T} \mathbf{f}_{\mathrm{int}}^{\mathrm{e}}
\end{aligned}
$$

$n l$ is the number of layers and $\zeta_{i}$ is the transverse reference coordinate of the $i$ th layer along the cross section of the element. The internal force vector $\mathbf{f}_{\text {int }}^{\mathrm{e}}$ can be obtained explicitly using compatible and enhanced strains: 


$$
\mathbf{f}_{\text {int }}^{\mathrm{e}}=\mathbf{f}_{\text {int }}^{\mathrm{u}}-\frac{f_{\alpha}}{k_{\alpha \alpha}} \mathbf{k}_{\alpha u}
$$

where $f_{\alpha}$ is given by:

$$
f_{\alpha}=\int_{V^{0}} \tilde{\mathbf{B}}_{\alpha} \mathbf{S} \mathrm{d} V
$$

where $\tilde{\mathbf{B}}_{\alpha}=\mathbf{T}_{0} \mathbf{B}_{\alpha}, \quad \mathbf{B}_{\alpha}=<\begin{array}{llllllll}0 & 0 & \zeta & 0 & 0 & 0\end{array}>^{T}$ and $\mathbf{T}_{0}$ are the curvilinear transformation matrix. $\mathbf{f}_{\text {int }}^{\text {e }}$

The global internal force vector $\mathbf{F}$ is then obtained by assembling element internal forces

$$
\mathbf{F}=\sum_{e=1}^{\text {nelt }} \mathbf{f}_{\text {int }}^{\mathrm{e}}
$$

The use of a full integration in the in-plane of the shell element, through the use of $2 \times 2$ Gauss points, allows the obtention of a stiffness matrix that has a correct rank, this reveals that the element has no hourglass modes; therefore, stabilisation is not required in the present model.

\section{Numerical applications}

\subsection{Validation of the model on necking prediction of a cylindrical tensile bar}

For validation purpose only, we first present numerical predictions of the proposed MCK model on the necking of a cylindrical tensile bar. This example is considered as a standard benchmark since it has been studied extensively by several authors (Needleman, 1972; Tvergaard \& Needleman, 1984) by using either Gurson or GTN models.

The cylindrical tensile bar of $8 \mathrm{~cm}$ length and $2 \mathrm{~cm}$ diameter is given in Figure 4. It is made of a common material with Young's modulus $E=300 \mathrm{~N} / \mathrm{cm}^{2}$ and a Poisson's ratio $v=.3$. An isotropic hardening of the matrix has been considered through a power law of the form:

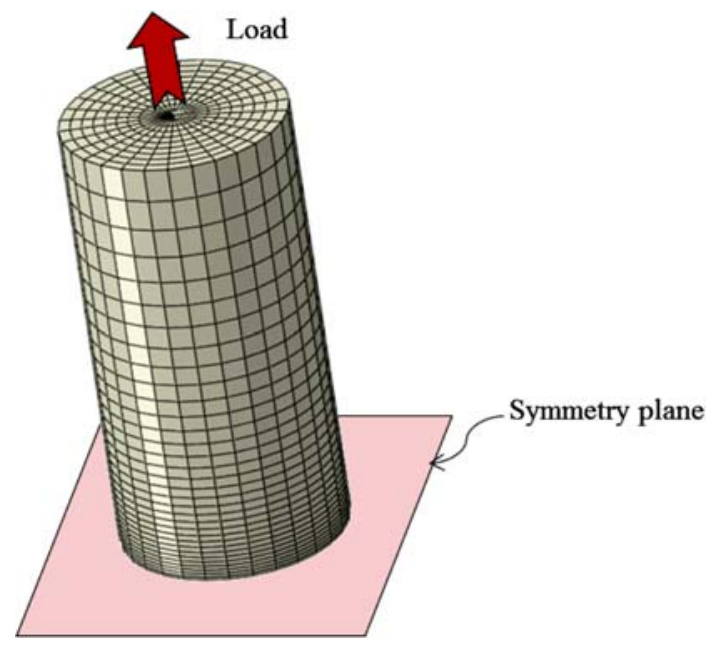

Figure 4. Finite element mesh of 1/2 cylindrical tensile test specimen. 


$$
\frac{\bar{\sigma}}{\sigma_{0}}=\left(\frac{\bar{\sigma}}{\sigma_{0}}+\frac{3 G}{\sigma_{0}} \bar{\varepsilon}_{m}^{p}\right)^{N}
$$

where $\sigma_{0}=1$ is the initial yield stress, $N=.1$ is the hardening parameter and $G$ is the elastic shear modulus.

As in Tveergard \& Needleman (1984), the initial porosity $f_{0}=0$ and the coalescence parameters $f_{F}=.6$ and $f_{\mathrm{c}}=.59$ have been adopted. The void nucleation parameters, $\varepsilon_{\mathrm{N}}=.3, s_{\mathrm{N}}=.1$ and $f_{\mathrm{N}}=.04$, used are the same as those given by Needleman (1972). For comparison with GTN model, we consider parameters $q_{1}=1.5, q_{2}=1$ and $q_{3}=2.25$. These parameters were introduced by Tvergaard (1981) to make the predictions of the Gurson model agree with numerical studies of an elastic-plastic medium containing a periodic array of voids.

The example problem focuses on the neck development, which is a precursor to failure in the form of cup-cone fracture. The formation of the neck results in a triaxial state of stress at the centre of the specimen, which accelerates the growth of the nucleated voids.

A finite element computation using our model implemented in LS-DYNA explicit dynamic code, using the 3D solid-shell mesh as illustrated in Figure 4. The specimen is fixed at the bottom edge and submitted to an imposed displacement to its top edge.

The predictions of nominal stress vs. the nominal strain of the present model and those obtained by the GTN model are given in Figure 5. It is shown that the maximum load is reached at a nominal strain of about $9 \%$, after which the specimen softens due to coalescence of voids and eventually fractures across the neck region. Due to the explicit nature of our calculation, we observed a relative influence of the dynamic loading speed on the final results. Different loading velocities have been tested, and finally a velocity of $.07 \mathrm{~m} / \mathrm{s}$ is found to be a good compromise between efficiency and speed.

Moreover, it is observed that the results predicted by the present model recover completely those of GTN model. In fact, it is well known that the stress triaxiality in the centre of the cylindrical tensile specimen is very low.

Figures 6 and 7 show total void volume fraction and pressure stress contours, respectively, at $11 \%$ for the two previous models. It appears that, for the porosity and for low stress triaxiality, the present model and the GTN one are in good agreement.

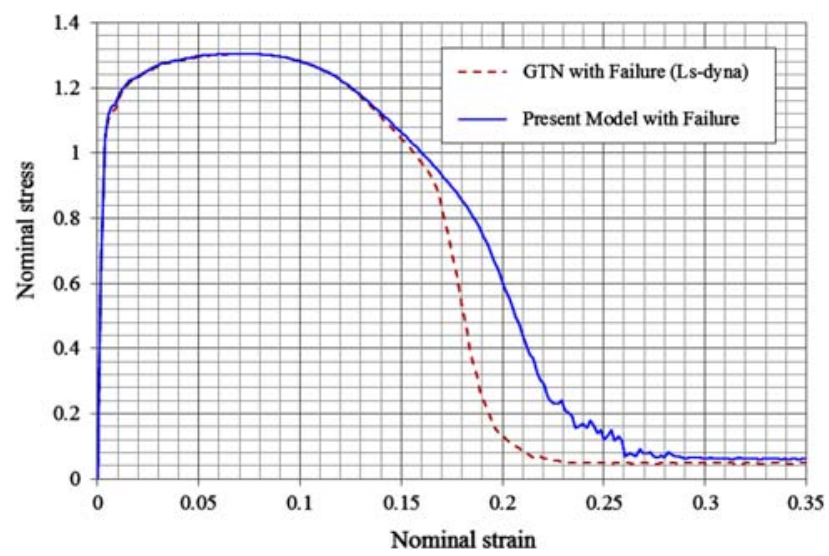

Figure 5. Stress-strain comparison between the present and the GTN models. 


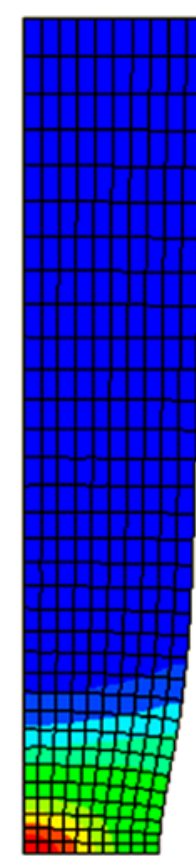

GTN model

$\left.\begin{array}{l}1.117 \mathrm{e}-01 \\ 1.005 \mathrm{e}-01 \\ 8.933 \mathrm{e}-02 \\ 7.817 \mathrm{e}-02 \\ 6.700 \mathrm{e}-02 \\ 5.583 \mathrm{e}-02 \\ 4.467 \mathrm{e}-02 \\ 3.350 \mathrm{e}-02 \\ 2.233 \mathrm{e}-02 \\ 1.117 \mathrm{e}-02 \\ 0.000 \mathrm{e}-00\end{array}\right]$

Figure 6. Void volume fraction distribution of the $1 / 4$ specimen at time $=9 \mathrm{~ms}$.

Present model

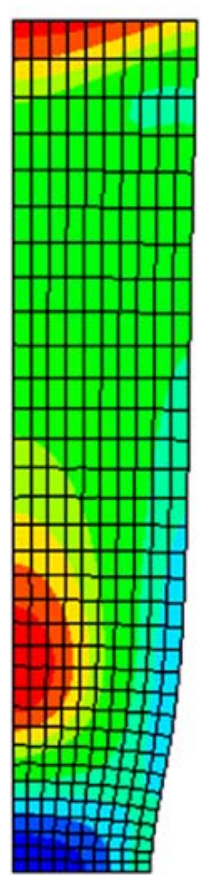

GTN model

$5.586 \mathrm{e}-01$
$3.902 \mathrm{e}-01$
$2.217 \mathrm{e}-01$
$5.330 \mathrm{e}-02$
$-1.151 \mathrm{e}-01$
$-2.836 \mathrm{e}-01$
$4.520 \mathrm{e}-01$
$-6.205 \mathrm{e}-01$
$-7.889 \mathrm{e}-01$
$-9.573 \mathrm{e}-01$
$-1.126 \mathrm{e}+00$

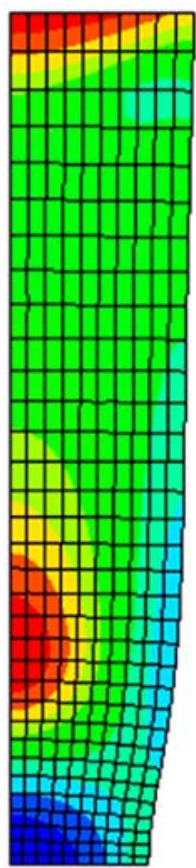

Present model
$7.207 e-02$

$6.486 \mathrm{e}-02$

$5.765 \mathrm{e}-02$

$5.045 \mathrm{e}-02$

$4.324 \mathrm{e}-02$

$3.603 \mathrm{e}-02$

$2.883 \mathrm{e}-02$

$2.162 e-02$

$1.441 \mathrm{e}-02$

$7.207 \mathrm{e}-03$

$0.000 \mathrm{e}+00$ 


\subsection{Modelling of trabecular bovine bone behaviour}

This section is devoted to the adaptation of our elastoplastic damage model to biological tissues by considering a basic cell in conformity with the trabecular bone microstructure. For this end, an estimate for the mechanical properties of the bone trabeculae can be identified by inverse methods using the experimental data of bovine trabecular bone described in Halgrin (2009).

In their previous work, Halgrin, Rahmoun, Chaari, Markiewicz, \& Drazetic, 2009 performed uniaxial compression tests of four cubic samples $10 \times 10 \times 10 \mathrm{~mm}^{3}$ extracted from the same bovine rib at a constant loading velocity of $10 \mathrm{~mm} / \mathrm{min}$ until the complete compaction of the sample.

Figure 8 shows the mechanical response of nominal stress against nominal strain for all four samples. We can observe that trabecular bone exhibits four different regimes, similar to those identified with other cellular materials (Gibson \& Ashby, 1997). A linear elastic behaviour is reached at low compression strains. Then, a peak stress occurs at strains of $10 \%$. After elastic buckling, the specimen seems to crush progressively with a quasi-constant stress. For strains higher than $60 \%$, the total compaction of the sample occurs and the compression force increases quickly, this last phase is known to be consolidation regime.

We conducted an inverse identification procedure to identify the bone matrix material parameters. It consists of fitting computed results and experimental measurements by finding the optimal set of constitutive parameters (Young's modulus $E_{\mathrm{m}}$ and the yield stress of the bone matrix $\bar{\sigma}$ ). The volume void fraction is measured by microtomography for each sample and the aspect ratio of spherical voids is fixed to $w=1$. The average value of volume void fraction based on experimental measurements using the microtomography is $77 \%$. It is important to notice that in the present formulation, the REV is not modelled classically using very fine mesh as reported by Halgrin et al. (2009). Therefore, thanks to the Mori-Tanaka scheme, the REV is modelled using a simple mapped mesh of only 1000 solid-shell elements (see Figure 9).

Table 1 summaries the obtained results using an inverse identification procedure. As we can observe, the identified Young's modulus of the bovine bone matrix $E_{\mathrm{m}}$ shows slight variations from $1700 \mathrm{MPa}$ to $2000 \mathrm{MPa}$, this is a direct consequence of the variations observed on the measurements data themselves. The same conclusions could be drawn, when observing the identified yield stress of the bone matrix $\bar{\sigma}$, which varies from $30 \mathrm{MPa}$ to $40 \mathrm{MPa}$. Finally,

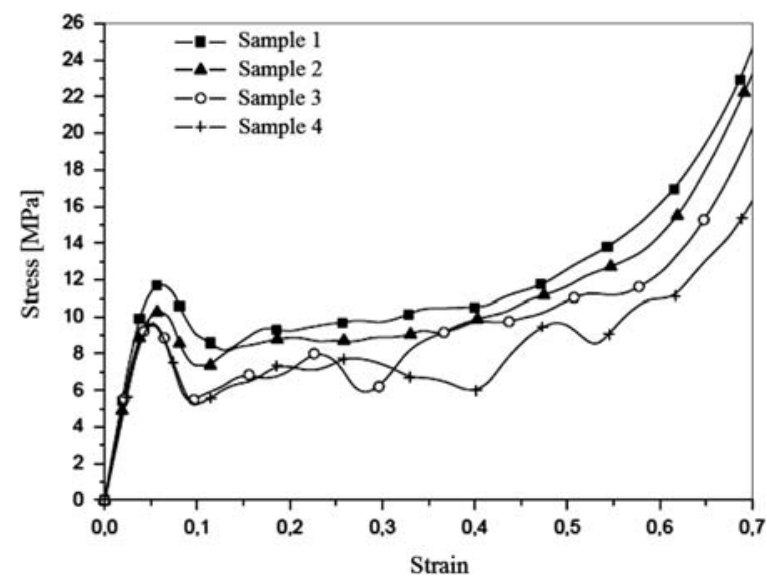

Figure 8. Experimental results of the four bovine rib samples under compression. 


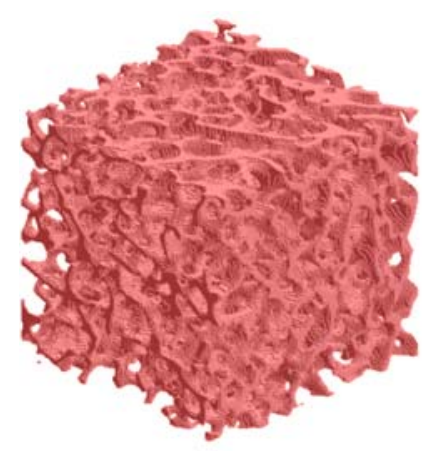

(a)

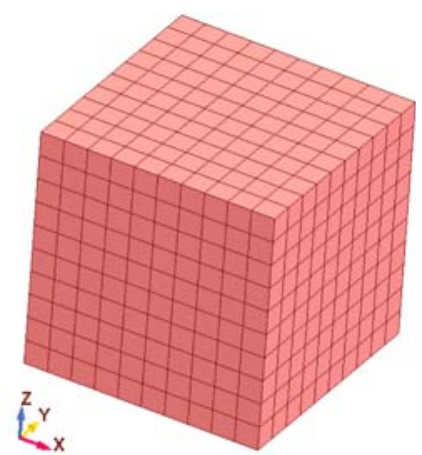

(b)

Figure 9. FE model: (a) REV classical fine mesh (424707 Hex elements in Halgrin et al., 2009) and (b) Present model $(10 \times 10 \times 10$ solid-shell elements $)$.

Table 1. Trabecular bovine bone material parameters.

\begin{tabular}{lccccc}
\hline & Sample 1 & Sample 2 & Sample 3 & Sample 4 & Min-max \\
\hline$E_{\mathrm{m}}(\mathrm{MPa})$ & 2000 & 1700 & 1700 & 1700 & $1700-2000$ \\
$\bar{\sigma}(\mathrm{MPa})$ & 40 & 38 & 33 & 30 & $30-40$ \\
$E_{\text {app }}(\mathrm{MPa})$ & 279.82 & 240.64 & 238.51 & 236.68 & $236.68-279.82$ \\
\hline
\end{tabular}

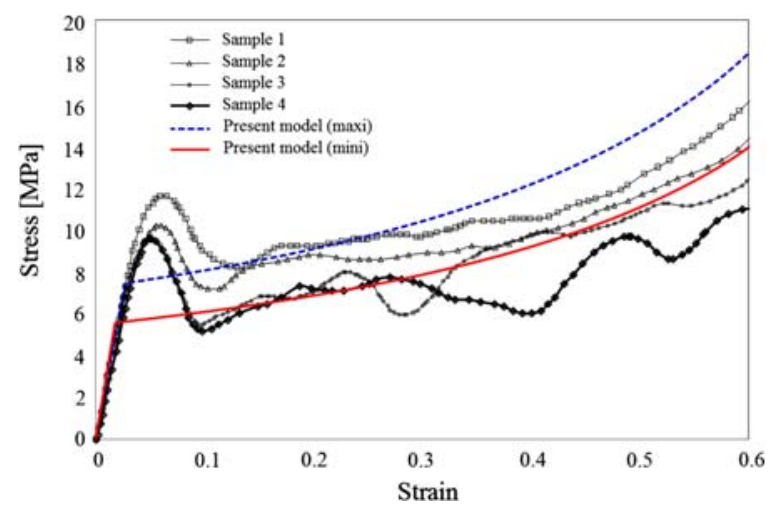

Figure 10. Numerical vs. experimental results of bovine trabecular bone behavior under compression.

thanks to the homogenised material tensor obtained, using the Mori-Tanaka scheme one can obtain the apparent Young's modulus $E_{\text {app }}$, which is usually used in biomechanical applications. These results are also presented in Figure 10.

\section{Conclusion}

In conclusion, the framework of homogenisation was used to derive a yield criterion using an approximate limit-analysis based on the MCK criterion for porous materials. The elastic properties of these materials were determined by a coupling from the Mori-Tanaka scheme and experimental measurements of anisotropy using microtomography techniques. The 
obtained model matches correctly the results obtained by the GTN model via a cylindrical tensile test specimen and allows to identify the mechanical response of trabecular bone. Its applications to the human femur bone are the subject of a forthcoming work.

\section{Acknowledgements}

The authors are grateful for support from the French institute of science and technology for transport, development and networks (IFSTTAR) under Masters Grant.

\section{References}

Carter, D.R., \& Hayes, W.C. (1977). The compressive behaviour of bone as a two phase porous structure. Journal of Bone \& Joint Surgery, 59, 954-962.

Charlebois, M., Jirásek, M., Philippe, K., \& Zysset, P.K. (2010). A nonlocal constitutive model for trabecular bone softening in compression. Biomechanics and Modeling in Mechanobiology, 9, 597-611.

Cowin, S.C. (1985). The relationship between the elasticity tensor and the fabric tensor. Mechanics of Materials, 4, 137-147.

Eshelby, J.D. (1957). The elastic field outside an ellipsoidal inclusion. Proceedings of the Royal Society London, 252, 561-569.

Eshelby, J.D. (1957). The determination of the elastic field of an ellipsoidal inclusion, and related problems. Proceedings of the Royal Society, Series A, 252, 561-569.

Fondrk, M.T., Bahniuk, E.H., \& Davy, D.T. (1999a). A damage model for nonlinear tensile behavior of cortical bone. Journal of Biomechanical Engineering, 121(5), 533-541.

Fondrk, M.T., Bahniuk, E.H., \& Davy, D.T. (1999b). Inelastic strain accumulation in cortical bone during rapid transient tensile loading. Journal of Biomechanical Engineering, 121(6), 616-621.

Fyhrie, D.P., \& Schaffler, M.B. (1994). Failure mechanisms in human vertebral cancellous bone. Bone, $15,105-109$.

Gibson. J. \& Ashby, M. 1997. Cellular solids: Structure and properties, 2nd ed. Cambridge, UK: Cambridge University Press.

Goulet, R.W., Goldstein, S.A., Ciarelli, M.J., Kuhn, J.L., Brown, M.B., \& Feldkamp, L.A. (1994). The relationship between the structural and orthogonal compressive properties of trabecular bone. Journal of Biomechanics, 27(4), 375-389.

Grassl, P., \& Jirásek, M. (2006). Plastic model with non-local damage applied to concrete. International Journal for Numerical and Analytical Methods in Geomechanics, 30(1), 71-90.

Gupta, A., Bayraktar, H.H., Fox, J.C., Keaveny, T.M., \& Papadopoulos, P. (2007). Constitutive modeling and algorithmic implementation of a plasticity-like model for trabecular bone structures. Computational Mechanics, 40, 261-272.

Gurson, A.L. (1977). Continuum theory of ductile rupture by void nucleation and growth: Part I. Yield criterion and flow rules for porous ductile media. Journal of Engineering Materials and Technology, 99, 2-15.

Halgrin, J. (2009). Effects of architectural parameters on the mechanical behavior of the trabecular bone, (in French) (PhD thesis), University of Valenciennes.

Halgrin J., Rahmoun J., Chaari F., Markiewicz, E., \& Drazetic P. (2009). Effets de l'architecture cellulaire de l'os spongieux sur sa réponse mécanique, 19 ême Congrès Francéais de Mécanique, Marseille, 24-28 août, France.

Hallquist, J.O. (2001). Ls-dyna Keyword User Manual, Livermore Software Technology Corporation.

Hannachi, M., Naceur, H., Batoz, J.L. (2007). Continuum based solid-shell element modeling for the optimization of composite multilayered structures. International Review of Mechanical Engineering. 1(4), 150-163.

Hansen, N.R., \& Schreyer, H.L. (1994). A thermodynamically consistent framework for theories of elastoplasticity coupled with damage. International Journal of Solids and Structures, 33(3), 359-389.

Harrigan, T.P., \& Mann, R.W. (1984). Characterization of micro structural anisotropy in orthotropic materials using a second rank tensor. Journal of Material Science, 19, 761-767.

Hayes, W.C., \& Carter, D.R. (1976). Postyield behavior of subchondral trabecular bone. Journal of Biomedical Materials Research, 10(4), 537-544.

Hill, R. (1965). The biochemists' green mansions: The photosynthetic electron transport chain. 19 Essays in Biochemistry, 1, 121-151. 
Hodgskinson, R., \& Currey, J.D. (1990). The effect of variation in structure on the young's modulus of cancellous bone: A comparison of human and non-human material. Proceedings of the Institution of Mechanical Engineers, 204, 115-121.

Kachanov, M., Shafiro, B., \& Tsukrov, I. (2003). Handbook of elasticity solutions. Dordrecht: Kluwer Academic.

Keaveny, T.M., Morgan, E.F., Niebur, G.L., \& Yeh, O.C. (2001). Biomechanics of trabecular bone. Annual Review of Biomedical Engineering, 3, 307-333.

Keaveny, T.M., Pinilla, T.P., Crawford, R.P., Kopperdahl, D.L., \& Lou, A. (1997). Systematic and random errors in compression trabecular bone. Journal of Orthopaedic Research, 15, 101-110.

Kefalas, V., \& Eftaxiopoulos, D.A. (2012). Experimental study of cancellous bone under large strains and a constitutive probabilistic model. Journal of the mechanical behavior of biomedical materials, $6,41-52$.

Kosmopoulos, V., Schizas, C., \& Keller, T.S. (2008). Modeling the onset and propagation of trabecular bone microdamage during lowcycle fatigue. Journal of Biomechanics, 41, 515-522.

Laws, N., \& McLaughlin, R. (1979). The effect of fibre length on the overall moduli of composite materials. Journal of the Mechanics and Physics of Solids, 27, 1-13.

Lubarda, V.A., \& Krajcinovic, D. (1995). Some fundamental issues in rate theory of damageelastoplasticity. International Journal of Plasticity, 11(7), 763-797.

Maugin, G.A. (1992). The thermomechanics of plasticity and fracture. Cambridge: Cambridge University Press.

Monchiet, V., Charkaluk, Kondo E., \& Kondo, D. (2007). An improvement of Gursontype models of porous materials by using Eshelby-like trial velocity fields. C.R. Mécanique, 335, 32-41.

Monchiet, V., Charkaluk, Kondo E., \& Kondo, D. (2011). A micromechanics-based modification of the Gurson criterion by using Eshelby-like velocity fields. European journal of mechanics A/Solids, 30, 940-949.

Mori, T., \& Tanaka, K. (1973). Averages stress in matrix and average elastic energy of materials with misfitting inclusions. Acta Metallia, 21, 571-574.

Mura, T. (1987). Micromechanics of defects in solids (2nd ed.). Boston, MA: Martinus Nijhoff, The Hague.

Needleman, A. (1972). A numerical study of necking in circular cylindrical bars. Journal of the Mechanics and Physics of Solids, 20, 111-127.

Rahmoun, J., Chaari, F., Markiewicz, E., \& Drazetic, P. (2009). Micromechanical modeling of the anisotropy of elastic biological composites. Multiscale Modeling and Simulation, 8(1), 326-33.

Rice, J.C., Cowin, S.C., \& Bowman, J.A. (1988). On the dependence of the elasticity and strength of cancellous bone on apparent density. Journal of Biomechanics, 21(2), 155-168.

Rincón-Kohli, L. 2003. Identification of a multiaxial failure criterion for human trabecular bone, (Doctoral dissertation, Ecole Polytechnique Fédérale de Lausanne).

Thurner, P.J., Wyss, P., Voide, R., Stauber, M., Stampanoni, M., Sennhauser, U., \& Méller, R. (2006). Time-lapsed investigation of three-dimensional failure and damage accumulation in trabecular bone using synchrotron light. Bone, 39, 289-299.

Tvergaard, V. (1981). Influence of voids on shear band instabilities under plane strain conditions. International Journal of Fracture, 17, 89-407.

Tvergaard, V., \& Needleman, A. (1984). Analysis of cup-cone fracture in round tensile bar. Acta Metallurgica, 32, 157-169.

Whitehouse, W.J. (1974). The quantitative morphology of anisotropic trabecular bone. Journal of Microscopy, 101, 153-168.

Zysset, P.K., \& Curnier, A. (1996). A 3D damage model for trabecular bone based on fabric tensors. Journal of Biomechanics, 29, 1549-1558. 